\title{
IN-VEHICLE NAVIGATION SYSTEMS: INTERFACE CHARACTERISTICS AND INDUSTRY TRENDS
}

\author{
Robert E. Llaneras and Jeremiah P. Singer \\ Westat \\ 1650 Research Blvd. \\ Rockville, MD 20850 \\ E-mail: 1lanere1@westat.com
}

\begin{abstract}
Summary: A review and inventory of in-vehicle navigation systems was conducted in order to better understand the current state of practice and trends relating to their design and implementation. The review focused on human factors characteristics and interface features using accepted human factors practices, principles, and guidelines as a basis for assessing likely impacts on driver distraction. The inventory examined market-ready in-vehicle products, and identified a range of interface design features, noting aspects and dimensions that have implications for potential driver distraction. Results indicated that devices tend to incorporate a large number of features and options, making it a potential challenge for drivers to learn all of the capabilities of a system and resulting in lengthy manuals. Although devices also tended to provide large amounts of information, some designs may allow for increased information presentation without necessarily sacrificing performance. Warnings or cautions against interacting with systems while driving were common; however, relatively few systems disable equipment when vehicles are in operation.
\end{abstract}

\section{INTRODUCTION}

The number of new vehicles offering navigation systems as standard or optional equipment in the U.S. has steadily increased within the past several years, with systems being offered in 54 model 2002 vehicles (J.D. Power, 2001). JD Power estimates that navigation systems will be available on 30 percent of all new vehicles by 2006, and six percent of new vehicle buyers will purchase these devices. Consumer satisfaction with navigation systems is strongly related not only to operational aspects of these systems (speed and accuracy), but with their ease-of-use as well (J.D. Power, 2000). User-friendly designs are especially important since the majority of consumers purchasing navigation systems are first-time users with no experience operating these types of complex devices.

\section{Purpose and Method}

An inventory of current in-vehicle technologies (navigation systems) was conducted in order to measure and document the range of interface design characteristics, noting aspects and dimensions that have implications for potential driver distraction. Twenty-eight navigation systems were identified in our review of available systems. Thirteen of these systems were subjected to in-depth reviews using a standardized data collection form to inventory basic interface design characteristics and features. Items in the form were developed from available human factors guidelines documents and accepted engineering principles, and were intended to 
capture safety-relevant design aspects and parameters. The form provided for the collection of an extensive amount of information (over 150 individual parameters), including display and control characteristics (type, location, legibility, number of menus, etc.); type of interaction modes (auditory, visual, haptic, etc.); and device interlocks or design restrictions, among other aspects.

\section{RESULTS}

Since drivers may devote significant attention to searching for and manipulating in-vehicle controls, reducing and simplifying the number of controls in the vehicle cockpit is generally viewed as a desirable characteristic. Overall, across all of the navigation systems reviewed (excluding the Rand McNally unit), devices averaged slightly over 11 hard controls (11.53) located on the system units. Systems furnished by OEMs tended to have fewer controls (8.80) than aftermarket suppliers (10.85). Navigation systems with touch screen interfaces (e.g., Honda, Lexus, Pathfinder, TravRoute) tended to have the fewest number of physical hard controls, ranging from four to seven controls.

All of the systems inventoried included some form of visual display which drivers could use to program destinations, view maps, receive system status information, access visual-based routing and guidance information, and perform other navigation-related tasks. The vast majority of systems $(80 \%)$ located their visual display in the center stack area of the instrument panel where conventional radio and HVAC controls are traditionally found. A few systems positioned the display on the dash area over the center stack; controls for these types of systems tended to be located in the center stack area. The Infinity system used this type of configuration, which featured a retractable display that is only visible when the system is in operation. This design positions the display closer to the driver's line of sight, yet limits distraction when the system is not in operation. Other manufactures also offer retractable displays, but not all are necessarily located along the top of the dashboard. A few manufacturers mount or position displays low in the vehicle cab near the floor console. All systems allowed the display to be easily viewed by a front seat passenger.

\section{Type and Amount of Information}

Most navigation systems rely on three primary guidance display screens to communicate navigation information to drivers: (1) maps, (2) maneuver lists with sequenced turn directions, and (3) turn-by-turn guidance displays which generally "pop up" in advance of a turn. The overall utility of each depends on the particular task being performed. Maps, for example, can be effectively used to plan a route since they provide a pictorial representation of an area or region, while ordered lists of directions can limit information processing and lead to fast and accurate navigation performance. Systems tend to use or make available all three types of information displays. Figure 1 provides examples of each type of navigation guidance display. With the exception of the Blaupunkt and Rand McNally systems, which have very small monochrome screens, all of the systems provided some form of map display. Indeed, 67 percent of the systems reviewed used the map as the default display screen following destination entry (i.e., during active navigation). This suggests that designers perceive that drivers will use map-based information not only for trip planning purposes, but also during en-route navigation. Map displays contained the most information (an average of over ten items), and generally served as the default guidance view (the one drivers would see first when operating the system) despite the 
fact that they rely heavily on spatial processing capabilities of the driver and tend to be a poor presentation method when the vehicle is moving. Most systems, however, provided supplemental turn-by-turn guidance displays. Systems also enabled drivers to change the default view, and/or access alternative views directly (often via a single button press).
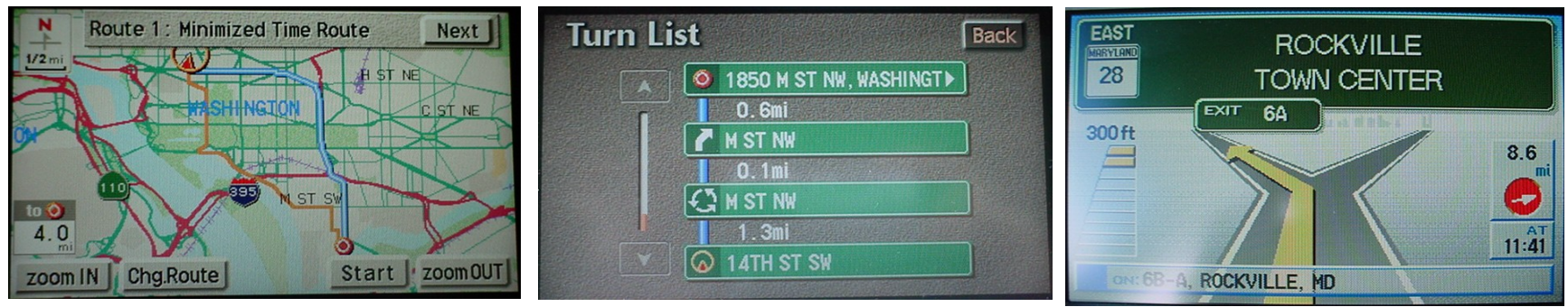

Figure 1. Map, Maneuver List, and Turn-by-Turn Display Screen Examples

Some systems also allowed the display screen to be blanked-out while driving, allowing drivers to receive audio guidance only. Although turn-by-turn guidance displays generally contained fewer information items than map displays, they still presented a considerable amount of information, averaging 8.5 information elements. General types of information on these displays tended to include turn arrows, countdown bars to indicate the distance to the next maneuver, an indication of the current and next turn street name, and an abbreviated map depicting the intersection roadway geometry at the next maneuver point. One interesting design adopted by several systems was to integrate display elements such as the turn arrow, countdown bar, map, and vehicle position indicator. This arrangement allows drivers to focus on a single overall graphic representation rather than several individual display elements to extract the information (exercising Gestalt principles). Another characteristic common to turn-by-turn displays was the redundant audio component announcing the upcoming maneuver. Some systems only announced the direction and distance of the next turn, rather than the specific street name. Exceptions were the Infinity, Nissan, Alpine, Lexus, Honda, and Garmin systems, which provided some road name outputs. The display type with the least resident amount of information was the maneuver list view (averaging about six elements). This view provided drivers with as few as two items of information in some cases, but generally provided turn arrows to indicate the direction, the street name, and distance to the next turn, as well as display elements (e.g., scroll bar).

\section{Destination Entry: Methods, Steps and Button Presses}

Our review documented the number of steps and button presses required to complete destination entry interactions using three basic destination entry methods: street address (100 Main Street, Rockville, MD), point of interest (closest Starbucks Coffee), and address book (select a previously entered destination). The process documented the necessary number of button presses (both minimum and maximum) required to complete the task assuming error-free performance. Thus, our analysis of the number of keystrokes or button presses required to complete typical destination entry tasks should be considered a conservative estimate. Since any given input method tended to have shortcuts (e.g., use of default or saved values, quick-spell, etc.) our inventory noted both the minimum and maximum number of key presses for each method.

Figure 2 illustrates the mean minimum and maximum number of button presses required to program a destination across all systems for each of three methods. As a group, destination entry 
tasks in our sample of navigation systems averaged from as few as six keystrokes to nearly 33 keystrokes. Individual systems with the fewest keystrokes in our sample required three to four keystrokes, and those with the longest required upwards of 50 button presses. In general, destination entry via address book required the least number of button presses, while entry using street address tended to require the most button presses. Compared to street address entry, for example, selecting a destination via an address book required half as many key/button presses under the best of circumstances. Differences in the number of steps or operations across methods accounted for differences in the observed number of key/button presses; as the number of steps increased, so too did the number of key presses. Destination entry using the address book to select a previously entered destination tended to have the fewest number of steps (averaging four to five), while both POI and street address required drivers to complete an average of six to eight operations.

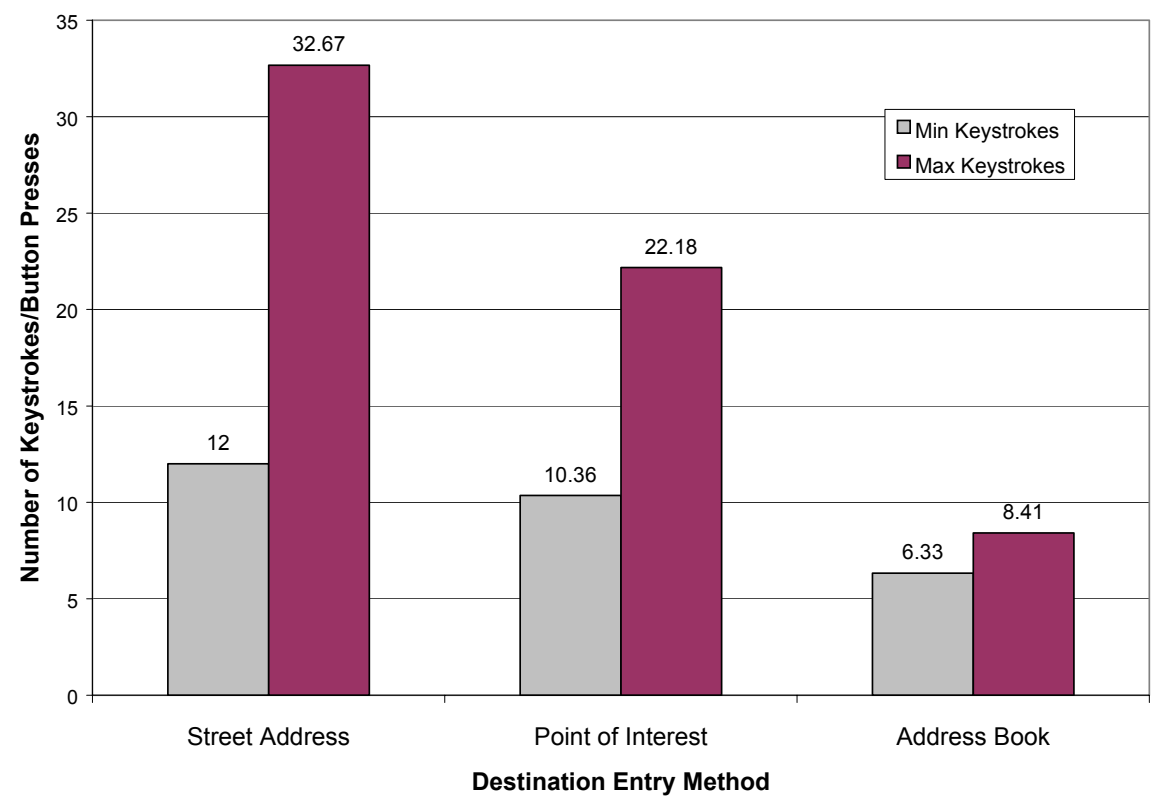

Figure 2. Navigation System Destination Entry: Mean Minimum and Maximum Key/Button Presses as a Function of Destination Entry Method

\section{Safety Features}

Almost all of the navigation devices included some form of warning or caution against use while driving; these specifically targeted significant interactions with the unit such as programming a destination. Warnings were incorporated into product start-up screens, user manuals, and sometimes integrated so that advisory messages are displayed when attempting to access a function while driving. Warnings tended to include most of the basic elements believed to contribute to effective warnings, including: (1) identification of the hazard, (2) consequences of ignoring the warning, and (3) instructions on how to avoid or control the hazard. Although many systems adhered to these basic principles, it is unlikely that warnings alone will dissuade all drivers from performing complex task interactions while the vehicle is moving. Lockouts, which prevent drivers from attempting complex task interactions while the vehicle is in motion, represent more proactive methods. Mandatory restrictions and lockouts to complex functions 
while driving was not a commonly adopted strategy, with less than one in five systems incorporating such features.

Programming a destination into a navigation system is arguably one of the most complex tasks drivers will perform with a telematics device. Concern over these types of multi-step and potentially demanding tasks while driving has spawned the development of several guidelines and recommended industry practices, both in the U.S. and abroad. Despite the complexity of destination entry tasks, many commercially available systems do not lock out destination entry while the vehicle is moving. Of the 12 passenger car systems examined as part of this study (excluding the Rand MacNally device), only three restrict the driver from accessing destination entry functions when the vehicle is in motion. Furthermore, only one of the systems examined (Lexus) supported voice-based destination entry, and even this system limited destination entry via voice input to the selection of previously defined destinations (e.g., "go home," "go to previous destination," etc.). All of the other systems required visual and manual interactions to program destinations and execute other task interactions such as modifying routes. Almost all of the systems reviewed support at least five different methods, with street address, point of interest, and address book entry methods among the most prevalent. In general, destination entry via address book required the least number of button presses, while entry using street address tended to require the most button presses.

\section{SUMMARY \& DISCUSSION}

All of the navigation systems inventoried (with the exception of the speech-based AudioNav) included some form of visual display. The location, size, and quality of these displays tended to conform to accepted human factors practices, but considerable variation exists, particularly for aftermarket systems. In general, no standard configuration for displaying screen elements or for organizing screen items was apparent. The number of physical controls present in our sample of navigation system is not out of line with conventional radio controls, averaging slightly over eleven. Despite current human factors guidelines, several OEM and many aftermarket navigation systems do not restrict or lock out complex tasks (e.g., destination entry) when the vehicle is moving. All warn the driver against attempting to interact with the device while driving, but few actually physically restrict access to demanding functions when the vehicle is in motion. Nevertheless, many systems do incorporate features that may minimize glance times to displays and manage information flow, such as limiting the number of available menu options or rows of items on a display and use of auditory outputs for routing information and system feedback. Systems also provide a wide variety of destination entry input methods, some requiring only several keystrokes to execute. Devices incorporate a relatively large number of features and options for configuring displayed information and executing tasks. As a consequence, manuals were voluminous, ranging from 41 to 145 pages, with most systems averaging about 80 pages.

\section{Trends and Common Practices in Interface Designs}

The following features and characteristics were frequently noted in our sample of navigation devices, and while they may not necessarily constitute industry "trends," they point to areas of common practice: 


\section{Displays \& Controls}

- Systems generally use color displays.

- There was no apparent standard configuration for displaying screen elements or for organizing on-screen items and controls for data entry. Most systems maintained adequate consistency between various displays, screen elements, and controls, but no common framework emerged across manufacturers.

- The ability to display split-screen views (e.g., map view and turn-by-turn, map view and turn list, etc.) appears to be a common feature in many navigation systems.

- Most systems possessed dedicated hard controls for frequently used/accessed functions (e.g., repeat system message). Many systems used re-configurable keys/buttons whose function change depending on the application.

\section{$\underline{\text { Auditory Displays/Interactions }}$}

- Manufacturers appeared to recognize that voice commands can be distracting or annoying, and at least in some cases, exercised care in selecting or designing for the type of transactions executed using voice commands. The Lexus navigation system, for example, limited the functions that were accessible in this manner.

- Use of a switch to activate the voice recognition system may increase system reliability. Systems also tended to provide both auditory and visual feedback to confirm driver inputs and availability of the speech command.

- Some systems adopt a strategy to alert drivers through auditory tones/beeps in advance of the presentation of information, and/or changes in display status. This may limit the need for drivers to continuously monitor the system, or repeat system messages.

\section{$\underline{\text { Safety Features }}$}

- Warnings or cautions against interacting with systems while driving were common.

- Many systems mute or reduce the volume of the radio/CD system when issuing auditory commands/messages/alerts. At least one navigation system manufacturer perceived this feature may annoy drivers and opted for a different approach.

\section{Operational Characteristics}

- Systems tend to support multiple-input methods and approaches for completing tasks. These alternatives impact the number of operations and button/key presses required to complete tasks.

- Touchscreen displays generally required fewer interactions than non-touch screen interfaces. Touchscreen displays do, however, tend to have some disadvantages such as lack of tactile feedback.

- Systems provided for operation at night or low-light conditions.

- Many navigation systems do not announce the street name of the upcoming maneuver/turn, despite the potential advantage that this type of information may provide (e.g., reduce wrong turn errors) 
- Time-out functions were evident in some systems. These were used to pace driver voice command inputs as well as to time the duration of displayed information.

- The majority of navigation systems used map-based information as the default both during pre-trip planning and active en-route navigation.

\section{ACKNOWLEDGEMENTS}

This work was sponsored by The National Highway Traffic Safety Administration. The opinions, findings and conclusions expressed in this publication are those of the author(s) and not necessarily those of the Department of Transportation or the National Highway Traffic Safety Administration.

\section{REFERENCES}

J.D. Power and Associates. 2000. Navigation Usage and Satisfaction Study.

J.D. Power and Associates. 2001. Reports: In-Vehicle Navigation Systems Poised for Significant Growth. October 11 Press Release 